\title{
Newer Aspects in Phototherapy
}

\author{
Sekar $\mathrm{CS}^{1}$, Srinivas $\mathrm{CR}^{2}$
}

${ }^{1}$ Professor of Dermatology, Department of Dermatology, PSG Hospitals Coimbatore, ${ }^{2}$ Professor \& HOD, Department of Dermatology, PSG Hospitals Coimbatore

\begin{abstract}
The concept of phototherapy has undergone a lot of changes in the past 30 years. It started with the emergence of psoralen ultraviolet A (PUVA) for the treatment of psoriasis. The subsequent evolution of Narrow band UVB therapy, Targeted phototherapy, UVA1 therapy, Home phototherapy and the use of LED lamps have revolutionized the treatment of various skin diseases. This article briefly summarizes the advances made in the last few years in the field of phototherapy.

Key words: Phototherapy, Excimer laser, Targeted phototherapy, Home phototherapy, Newer lamps
\end{abstract}

\section{History}

A round the turn of the $19^{\text {th }}$ century, Sardemann used, for the first time ever, a carbon arc lamp to treat psoriasis in a patient. ${ }^{1}$ In 1923 , Alderson then described "heliotherapy in psoriasis". He used a quartz-jacketed mercury discharge lamp to treat his first patient. ${ }^{2}$ William Goeckermann, a dermatologist at the Mayo Clinic (Rochester, Minn) followed and introduced a combination of coal tar and subsequent UV-B, which soon came to fame as the "Goekermann regimen". ${ }^{3}$ In 1974, the combination of oral psoralen intake and subsequent UV-A irradiation was reported by Parrish ${ }^{4}$ and Wolff ${ }^{5}$. These publications marked the initiation of modern psoralen-UV-A (PUVA) photochemotherapy for psoriasis.

\section{Introduction}

Phototherapy (UV-A and UV-B) has become one of the most commonly used modalities of treatment of a variety of skin diseases, although the mechanism of action have not been fully understood. Inhibition of DNA synthesis by UV radiation is proposed as a mechanism in proliferating skin diseases.

Phototherapy exerts its therapeutic effect, via a number of mechanisms. The major molecular target

\footnotetext{
Address for correspondence

Dr. C Shanmuga Sekar

Professor,

Dept of Dermatology, PSG Hospitals

Coimbatore-641004

Telephone no-0422 2570170

E-mail:drshanmugasekar@gmail.com
}

for ultraviolet B (UVB) radiation is nuclear DNA which absorbs light, generating pyrimidine dimers and other photoproducts. This action ultimately inhibits DNA synthesis.

Psoralen ultraviolet A (PUVA) is the combined use of psoralen and long-wave UV radiation (UVA). One molecule of psoralen intercalates into the DNA double strand; upon irradiation with UVA, one photon of light is absorbed, followed by binding of a thymine base. The DNA-psoralen crosslink inhibits DNA replication and causes cell cycle arrest.

Narrow band UVB therapy using $311 \mathrm{~nm}$ is increasingly being used in a variety of skin disorders such as psoriasis and vitiligo.

Both UVA and UVB affect cytokine production of inflammatory cells, reducing the secretion of inflammatory cytokines, and also cause apoptosis of lymphocytes. ${ }^{6}$

\section{Newer aspects}

A lot of advances have been made in phototherapy since its emergence. The indications for phototherapy have expanded, newer machines and lamps have emerged and therapeutic protocols have been modified. This article will discuss briefly regarding the newer advances made in the field of phototherapy.

\section{Targeted phototherapy}

Targeted ultraviolet (UV) B phototherapy devices provide a practical means to treat localized lesions 
while sparing harmful effects to unaffected skin. ${ }^{7}$ The most common and extensively implemented mode of delivery is the 308-nm excimer laser. Although laser treatment is the dominant standard of 308-nm excimer delivery, numerous studies have reported the use of a 308-nm monochromatic excimer nonlaser (MEL) light source. UV light is also used as a source for targeted phototherapy.

\section{Excimer laser}

Excimer stands for excited dimer, a diatomic molecule usually of an inert gas atom and a halide atom, which are bound in excited states only. These diatomic molecules have extremely short lifetime and dissociate releasing the excitation energy through UV photons. These devices are xenon-chloride gas excimer lasers that deliver coherent, monochromatic UVB light of 308-nm wavelength in short impulses and allow for changes in important phototherapeutic parameters such as impulse frequency and light intensity. In addition to the treatment of psoriasis, excimer lasers can be used for treating vitiligo, atopic dermatitis, and leukoderma. ${ }^{8}$

Several advantages have been claimed for targeted phototherapy: ${ }^{9}$

a. Exposure of involved areas only and sparing of uninvolved areas, thus minimizing acute side effects such as erythema and long-term risk of skin cancer over unaffected skin.

b. Quick delivery of energy and thereby shortened duration of treatment.

c. Delivery of higher doses (super-erythemogenic doses) of energy because uninvolved areas are not exposed, higher doses of energy can be delivered selectively to the lesions, thereby enhancing efficacy and achieving faster response.

d. The maneuverable hand piece allows treatment of difficult areas such as scalp, nose, genitals, oral mucosa, ear, etc.

e. Easy administration for children as delivery is hand-held.

f. Targeted phototherapy machines occupy less space.

However, targeted phototherapy devices have certain disadvantages; they are more expensive. Also, they are not adequate to treat extensive areas in view of the cost of treatment and time involved in treatment. They are not recommended for use if lesions occur over more than $10 \%$ of the body area. High cost and difficulties in maintenance are the other disadvantages with this machine.

\section{Fractional targeted phototherapy}

Targeted phototherapy delivers a high amount of UV rays over a small area and is used for the treatment of smaller lesions and for lesions resistant to whole body phototherapy using UV chamber. Fractionalization of the UV light with the same irradiance to target smaller areas has the additional advantage of using fewer shots and also covering larger areas. Since the light is not collimated, it is reasonable to assume that the rays diverge following entry into skin and thus can target larger areas. ${ }^{10}$ Targeting very small areas may also minimize the chance of UV burn.

\section{Turban PUVASOL}

In the turban PUVASOL therapy, $1 \mathrm{ml}$ of 8-methoxypsoralen (8-MOP) 1\% is diluted in 2 liters of water. A clean absorbent cotton cloth is soaked into the solution for 30 seconds and wrapped around the scalp in a turban-like fashion for 5 minutes. The process was repeated four times. The patients were further exposed to sunlight for 5 minutes and the time was gradually increased up to 15 minutes. The treatment was carried out thrice a week. The time of exposure was between 10:00 and 10:15 AM when the ultraviolet UVA : UVB ratio is maximum. The patient was advised to avoid sun exposure for few hours after treatment. $A$ few reports indicate that the treatment is useful in the treatment of alopecia totalis. ${ }^{11}$

\section{UVA1}

Most recently, ultraviolet A1 (UVA1) phototherapy has emerged as a specific UVR phototherapeutic mechanism. High intensity long-wavelength ultraviolet A (340-400 nm; UVA1) lamps were initially developed as skin research tools; over time they have proven to be useful for treating a number of chronic dermatoses. ${ }^{12}$ UVA1 units and dosimetry are strikingly different from conventional UV phototherapy. The therapeutic effect of UVA1 is related to the fact that its long wavelength penetrates the dermis more deeply than UVB. UVA1 radiation induces collagenase (matrix metalloproteinase-1) expression, T-cell apoptosis, and depletes langerhans and mast cells in the dermis. UVA1 exposure stimulates endothelial cells to undergo neovascularization. Ultraviolet $A 1$ exerts significant therapeutic effects in atopic dermatitis and morphea; there is also evidence for its use in other skin diseases, including cutaneous T-cell lymphoma and mastocytosis. UVA1 therapy also has been shown to be relatively free of side effects associated with other phototherapy regimens, including erythema and 
cellular transformation. ${ }^{13}$ These properties make UVA1 phototherapy an important treatment option for many debilitating skin conditions.

\section{Home phototherapy}

Home phototherapy equipment became commercially available in the early 1980s. Since its introduction, the use of home phototherapy has expanded and is popular with many psoriasis patients. Units of varying sizes have been designed in treating different types of diseases in various anatomic locations and distribution. Smaller, specialized targeted units are useful to treat specific body areas and eliminate exposure of unaffected skin to ultraviolet light. The ability to perform such localized treatment may be more efficacious in treating hand and foot diseases that require more intense regional treatment to penetrate the thick stratum corneum. For patients with more diffuse or total-body disease, reflecting side panels and wings are used to increase the dispersion of ultraviolet rays, thus allowing a greater area to be well-treated in a uniform manner and in a lesser amount of time.

Prior to the initiation of a home phototherapy regimen, patients should undergo examination by their dermatologist to determine skin type and to establish diagnosis. Additionally, a previous therapeutic response to outpatient phototherapy should be documented. The patient should be thoroughly educated as to practical use issues and goals of phototherapy as well as the anticipated response to and possible sideeffects of treatment

A variety of protocols for home phototherapy have been developed and are tailored to fit different diseases, skin types, and minimal erythema dose (MED). ${ }^{14}$ A standard protocol for NB-UVB in the treatment of psoriasis consists of three sessions per week (treatment sessions every other day is most effective) for a duration of three months, with initial treatment time determined by skin type or MED and dose escalation depending on the patient's response to treatment and the physician/clinic preferences. For MED-based NB-UVB regimens, 0.7 MED is the most frequently used initial dose, with recommended range of 0.5 - 1.0 MED. ${ }^{15,16}$ In terms of increase in treatment time, the magnitude of dose increase depends on the patient and the provider. There are a variety of strategies to increase treatment time: some increase the dose in increments of 10-20 percent with each treatment session; others use larger dose increments $(15-20 \%)$ early in the course of treatment followed by smaller increments (10\%) later on. Whereas MEDbased therapy is thought to be safest, treatment time based on the patient's skin type is more convenient and as such is utilized by many practitioners. ${ }^{17}$

Adverse effects associated with phototherapy include both acute adverse effects such as erythema and burning sensation and cumulative, dose-related effects that occur with prolonged use. However, the existing data demonstrates no differences in acute adverse events or total cumulative ultraviolet dose and there are no data suggesting that home phototherapy is less safe than standard outpatient treatment. ${ }^{18}$

\section{Newer lamps}

One of the major technological breakthroughs in the last decade is represented by the diversified medical applications of light-emitting diodes (LEDs). LEDs emitting in the ultraviolet (UV) B spectrum might serve as a more convenient alternative for targeted delivery of phototherapy in inflammatory skin diseases such as psoriasis. Kemény et al. ${ }^{19}$ reported the use of UVB LEDs in the treatment of psoriasis. The device used in their study emits $1 \mathrm{~mW} \mathrm{~cm}^{-2}$ at a central wavelength of $310 \mathrm{~nm}$ with 15-nm bandwidth, near to the optimum wavelength for the treatment of psoriasis. They show that this device can treat small areas of psoriasis, as would be expected from such an output. UVA LED 20 sources are also now available with outputs in excess of $4000 \mathrm{~mW} \mathrm{~cm}^{-2}$

\section{Conclusion}

As new data emerge, the role of phototherapy in treating many newer diseases is emerging. The scope for phototherapy is broadening with a lot of research into various aspects and it may emerge as one of the most promising dermatotherapies in the future. 


\section{References}

1. Diffey BL, Farr PM. An appraisal of ultraviolet lamps used for the phototherapy of psoriasis. Br J Dermatol 1987; 117: 49-56. http://dx.doi. org/10.1111/j.1365-2133.1987.tb04090.x

2. Parrish JA. Ultraviolet phototherapy of psoriasis. Pharmacol Ther 1981; 15: 313-20. http:// dx.doi.org/10.1016/0163-7258(81)90047-4

3. Honigsmann H. Phototherapy for psoriasis. Clin Exp Dermatol 2001; 26: 343-50. http:// dx.doi.org/10.1046/j.1365-2230.2001.00828.x

4. Parrish JA, Fitzpatrick TB, Tanenbaum L, Pathak MA. Photochemotherapy of psoriasis with oral methoxsalen and longwave ultraviolet light. N Engl J Med 1974; 291: 1207-11. http://dx.doi. org/10.1056/NEJM197412052912301

5. Wolff K, Honigsmann H, Gschnait F, Konrad K. Photochemotherapy of psoriasis: clinical experiences with 152 patients (author's transl). Dtsch Med Wochenschr 1975; 100: 2471-7. http://dx.doi.org/10.1055/s-0028-1106568

6. Morita A, Krutmann J. Ultraviolet A radiationinduced apoptosis. Methods Enzymol 2000; 319: 302-9. http://dx.doi.org/10.1016/S00766879(00)19031-7

7. Mysore V. Targeted phototherapy. Indian J Dermatol Venereol Leprol 2009; 75: 119-25. http://dx.doi.org/10.4103/0378-6323.48655

8. Mudigonda T, Dabade TS, Feldman SR. A review of targeted ultraviolet B phototherapy for psoriasis. J Am Acad Dermatol 2012; 66: 664-672. http://dx.doi.org/10.1016/j. jaad.2011.07.011

9. Grimes PE. Advances in the treatment of vitiligo: Targeted phototherapy. Cosmet Dermatol 2003; 16: 18-22.

10. Shanmuga SC, Srinivas C R. Fractional-targeted phototherapy. Indian Dermatol Online J 2014; 5: 104-5. http://dx.doi.org/10.4103/22295178.146171.

11. Sornakumar L, Sekar C S, Srinivas C R. Turban PUVASOL: An effective treatment in alopecia totalis. Int J Trichol 2010; 2: 106-7. http:// dx.doi.org/10.4103/0974-7753.77520
12. York NR, Jacobe HT. UVA1 phototherapy: a review of mechanism and therapeutic application. Int J Dermatol 2010; 49: 62330. http://dx.doi.org/10.1111/j.13654632.2009.04427.x

13. Zandi S, Kalia S, Lui H. UVA1 phototherapy: a concise and practical review. Skin Therapy Lett. 2012; 17: 1-4.

14. Zanolli MD, Feldman SR. Phototherapy treatment protocols : for psoriasis and other phototherapy responsive dermatoses. 2 nd ed. New York: Informa Healthcare:2004.

15. Zanolli M. Phototherapy arsenal in the treatment of psoriasis. Dermatol Clin 2004; 22: 397-406. http://dx.doi.org/10.1016/j. det.2003.12.003

16. Valkova S. UVB phototherapeutic modalities. Comparison of two treatments for chronic plaque psoriasis. Acta Dermatovenerol Alp Panonica Adriat 2007; 16(1): 26-30.

17. Schneider LA, Hinrichs R, Scharffetter-Kochanek K. Phototherapy and photochemotherapy. Clin Dermatol 2008; 26: 464-76. http://dx.doi. org/10.1016/j.clindermatol.2007.11.004

18. Koek MB, Buskens $E$, van Weelden $H$, Steegmans $\mathrm{PH}$, Bruijnzeel-Koomen CA, Sigurdsson V. Home versus outpatient ultraviolet $B$ phototherapy for mild to severe psoriasis: pragmatic multicentre randomised controlled noninferiority trial (PLUTO study). BMJ 2009; 338: 1542. http://dx.doi.org/10.1136/bmj.b1542

19. Kemény L, Csoma $Z$, Bagdi $E$, Banham $A H$, Krenács L, Koreck A. Targeted phototherapy of plaque-type psoriasis using ultraviolet B-light-emitting diodes. $\mathrm{Br} J$ Dermatol 2010; 163: 167-73. http://dx.doi.org/10.1111/ j.1365-2133.2010.09763.x

20. Edwards C, Anstey AV. Therapeutic ultraviolet light-emitting diode sources: a new phase in the evolution of phototherapy. $\mathrm{Br} J$ Dermatol 2010; 163: 3-4. http://dx.doi.org/10.1111/ j.1365-2133.2010.09870.x 